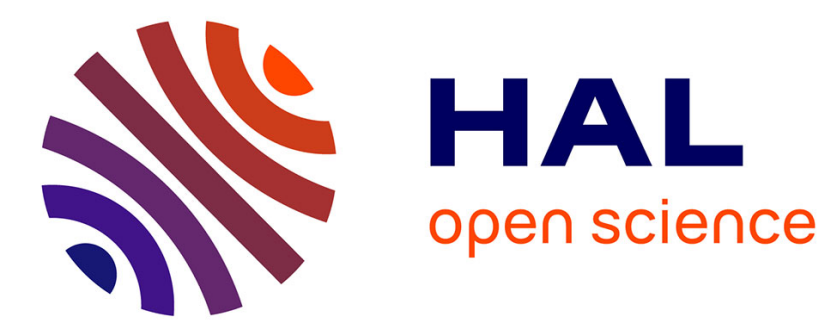

\title{
Pure longitudinal plate mode excited by poled domains transducers on LiNbO3
}

\author{
E. Courjon, J. Masson, D. Gachon, L. Gauthier-Manuel, N. Bodin, W. \\ Daniau, S. Ballandras, J. Hauden
}

\section{- To cite this version:}

E. Courjon, J. Masson, D. Gachon, L. Gauthier-Manuel, N. Bodin, et al.. Pure longitudinal plate mode excited by poled domains transducers on LiNbO3. EFTF-FCS'07, 2007, Geneve, Switzerland. pp.1073-1076, 10.1109/FREQ.2007.4319244 . hal-00194787

\section{HAL Id: hal-00194787 \\ https://hal.science/hal-00194787}

Submitted on 20 Apr 2021

HAL is a multi-disciplinary open access archive for the deposit and dissemination of scientific research documents, whether they are published or not. The documents may come from teaching and research institutions in France or abroad, or from public or private research centers.
L'archive ouverte pluridisciplinaire HAL, est destinée au dépôt et à la diffusion de documents scientifiques de niveau recherche, publiés ou non, émanant des établissements d'enseignement et de recherche français ou étrangers, des laboratoires publics ou privés.

\section{(c)(1)}

Distributed under a Creative Commons Attribution| 4.0 International License 


\section{Pure longitudinal plate mode excited by poled domains transducers on $\mathrm{LiNbO} 3$}

\author{
E. Courjon, J. Masson, D. Gachon, L. Gauthier-Manuel, W. \\ Daniau, N. Bodin, S. Ballandras \\ Institut FEMTO-ST, UMR 6174 CNRS, UFC-ENSMM-UTBM \\ 32 Ave. de l'Observatoire, 25044 Besançon Cedex, France \\ sylvain.ballandras@femto-st.fr
}

\author{
J. Hauden, \\ Photline Technologies \\ Besançon, France \\ Jerome.hauden@photline.com
}

\begin{abstract}
In this paper, we present new results on the development of piezoelectric transducers based on periodically poled ferroelectrics domains. The fabrication of test devices operating in the range $50-500 \mathrm{MHz}$ has been achieved. Pure longitudinal modes are excited, yielding high frequency operation of test devices with easily achievable lithography process (20 and $10 \mu \mathrm{m}$ periods). The dispersion analysis of our devices is reported and the capability of the proposed waveguide to support pure longitudinal modes along the plate radial dimension is emphasized
\end{abstract}

\section{INTRODUCTION}

The possibility to manufacture elastic wave-guide based on a thin ferroelectrics film deposited on a single crystal substrate and periodically poled to enable the excitation of surface acoustic wave has been demonstrated recently [1]. The so-called periodically poled piezoelectric inter-digital transducer (PPT) simply consists in two electrically conductive media embracing a periodically poled ferroelectrics layer. It has been successfully implemented and tested for the excitation of elliptically polarized waves in the frequency range of 1-3 GHz.

In previous work, we have investigated the possibility to develop PPTs on $\mathrm{LiNbO}_{3}$ [2]. The first experiments have been performed on optical grade $500 \mu \mathrm{m}$ thick 3" Z-cut wafers. We used optical - instead of acoustics - grade wafers because of their tighter tolerances in terms of total thickness variation and side parallelism. The fabrication of PPTs on such wafers gives access to the excitation of symmetrical Lamb modes with an operating frequency twice higher than those obtained using standard inter-digital transducers.

In this paper, the fabrication of high frequency PPTs exhibiting poling periods of 10,20 and $40 \mu \mathrm{m}$ have been successfully realized $(5,10$ and $20 \mu \mathrm{m}$ line-width respectively). Several millimeter long ferroelectrics gratings of respectively 3960,1980 and 990 periods have been tested and the corresponding admittances have been measured. Contributions corresponding to the elliptically polarized Lamb

This work has been funded by the European Community as the InterReg III project DOMINOS. waves already observed for lower frequency devices have been identified, corresponding to a wave velocity close to $3800 \mathrm{~m} \cdot \mathrm{s}^{-1}$ (near the Rayleigh wave velocity). Also clear contributions corresponding to a higher phase velocity have been observed. Simulations have been achieved using our periodic finite element code, enabling to analyze the experimental responses. It was found that the abovementioned contributions correspond to a pure longitudinal wave homogeneously distributed along the whole thickness of the plate and propagating near $6500 \mathrm{~m} . \mathrm{s}^{-1}$. Such a wave cannot be excited using standard Inter-Digital Transducer (IDT). Consequently, the capability of the poled transducer to excite it allows for the implementation of very high frequency devices thanks to the combination of a high phase velocity and the natural operation of the transducer, doubling the operation frequency compared to standard IDT for a given spatial period. The paper reports on the theory/experiment assessment and conclude on the applicability of such a solution for high frequency signal processing.

\section{Periodically Poled TRansducer (PPT) PRINCIPLE}

The PPT is fundamentally based on a periodically poled piezoelectric medium. Each side of this medium is metallized, then providing a capacitive dipole in which elastic waves can be excited thanks to phase construction. Such a periodically poled structure can be advantageously achieved on ferroelectrics materials like PZT [1] because of the rather small value of its coercitive electric field (the absolute value of the electric field above which the ferroelectrics poling can be controlled).

The principal advantages of PPTs compared to standard periodic inter-digital electrode transducer (IDTs) deposited atop piezoelectric materials are first the robustness of the excitation versus defects or surface contamination (no passivation is required to avoid short circuit as for standard IDTs) and second the opportunity to excite waves exhibiting a wavelength equal to the poling period, contrarily to standard IDTs for which the wavelength is equal to twice the mechanical period (Bragg condition). One can also note that 
comparably to film bulk acoustic resonators (FBARs), it can operate with large excitation powers as the electrodes are separated by a bulk material layer. We have shown in [2] the capability of the structure to excite elliptically polarized plate waves (Lamb modes).

To improve the understanding of the device operation, the dispersion curves of Lamb modes on a Z-cut $\mathrm{LiNbO}_{3}$ are plotted in fig.1, allowing us to point out the operating frequency of the excited modes for each of our sample configurations [2]. A simple geometric construction gives the operating frequencies for each excited Lamb mode [3]. For a given plate thickness $h$, the slope $m$ of a straight line passing through the origin is equal to the wavelength-plate thickness ratio $(m=\lambda / h)$. In our case, the thickness of the wafer is 500 $\mu \mathrm{m}$, a standard value for commercially available material (e.g. Crystal Tech Inc.). We consider periods of our experimental setups (200 and $100 \mu \mathrm{m}$ respectively) yielding values of $m$ equal to 0.4 and 0.2 (our devices, the acoustic wavelength $\lambda$ equals the poling period $p$ ). Projecting the corresponding lines on the Lamb wave dispersion curves allows for the prediction of the wave velocity and hence of the operating frequency for each mode.

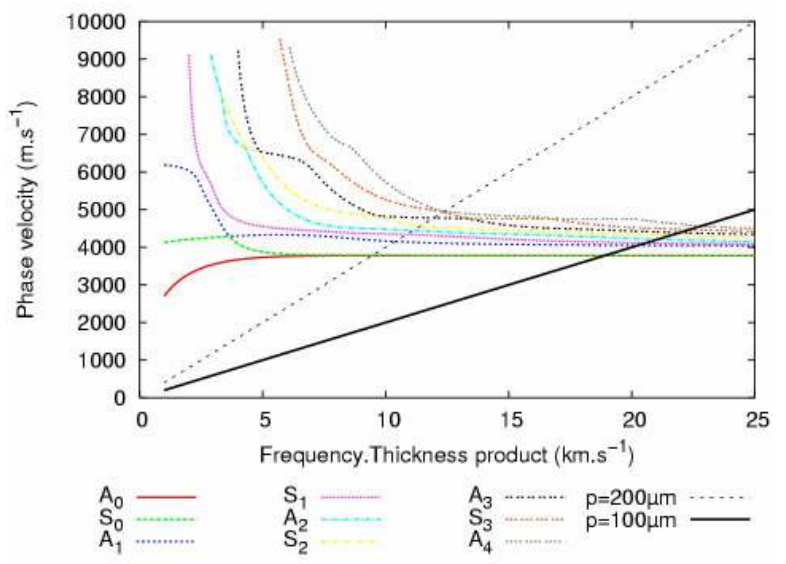

Figure 1. Dispersion curve of Lamb waves on a Z-cut $\mathrm{LiNbO}_{3}$ plate, frequency-thickness product ranging from 0 to $25 \mathrm{~km} \cdot \mathrm{s}^{-1}$. The straight lines indicate the expected operating rules of the experimental PPT-based devices

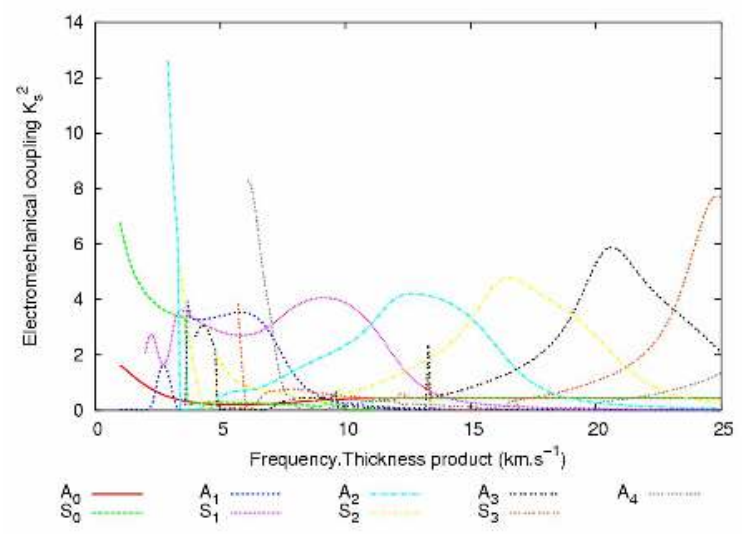

Figure 2. Coupling coefficients of the corresponding modes versus frequency.thickness product
Since the values of $m$ are always smaller than 1 (i.e. the frequency-thickness products corresponding to the test device are in the range $5-50 \mathrm{~km} . \mathrm{s}^{-1}$ ), we could not take advantage of the high velocity of the $S_{0}$ mode. As a consequence, the first coupled mode on our device should always exhibit a wave velocity close to $3750 \mathrm{~m} . \mathrm{s}-1$, yielding operating frequencies respectively equal to about 19 and $38 \mathrm{MHz}$. This first order prediction nicely meets the experimental values. Note that the corresponding experimental coupling coefficient generally remains moderate, between $1 \%$ and $1 \%$, whereas theoretical values are found in the range $1-10 \%$. This point needs more investigation to be clearly explained. More, note practical implementation only reveals the signature of symmetrical modes, assuming perfectly symmetrical transducer structures.

As previously presented [2], we have exploited a dedicated poling bench to control the poling of thick $(500 \mu \mathrm{m}) \mathrm{Z}$-cut $\mathrm{LiNbO}_{3}$ plates. It mainly consists in a high voltage amplifier used to apply an electric field to the $\mathrm{LiNbO}_{3}$ wafer strong enough to invert the spontaneous polarization of the material. The domains to be poled are defined thanks to a photo-resist pattern on top of a plate surface. This yields a dielectric barrier strong enough to prevent any electric field to be located underneath the resist. The high poling voltage is applied to the plate following the sequence shown in fig. 3 and using liquid electrodes made with a saturated lithium chloride solution. This sequence is designed to favor the domain nucleation, to stabilize the inverted domains (i.e. to avoid back-switching of the domains) and to avoid electrical breakdowns [4]. The poling process is monitored thanks to the measurement of the electric current crossing the wafer during the sequence. The signature of a successful domain inversion corresponds to the current discharge observed in fig.3. The poling can be easily controlled by a simple optical post-observation, as it generates a contrast between at the edge of the poled domains (see fig. 4).

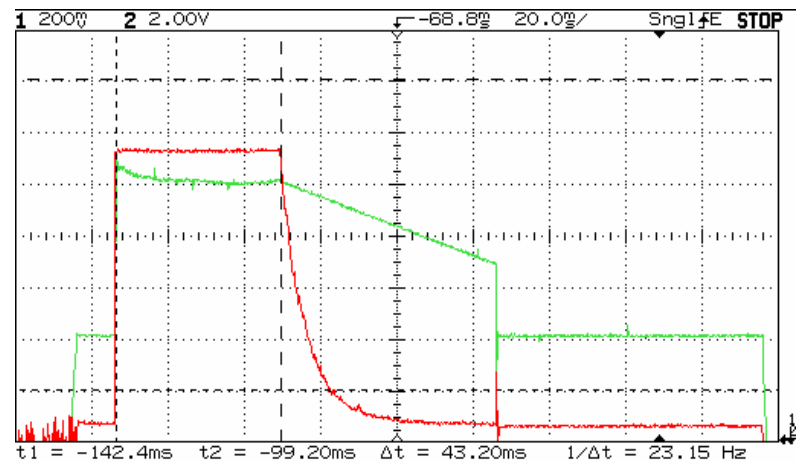

Figure 3. Domain inversion monitoring: probed high voltage applied on the wafer (green curve, poling sequence) and resulting crossing current (red curve). Voltage and current gain are respectively $1 \mathrm{~V} / 5000 \mathrm{~V}$ and $1 \mathrm{~V} / 5 \mathrm{~mA} .1^{\text {st }}$ step : the applied electric field is smaller than the coercitive limit, to prepare the sample for the nucleation step. $2^{\text {nd }}$ step : the applied field overcomes the coercitive limit, yielding nucleation and consequently poling (this step lies between the 2 dashed cursors). $3^{\text {rd }}$ step : linear decrease of the field to end-up the nucleation (self-termination step, exponential decrease of the current). $4^{\text {th }}$ step : stabilization of the poling by applying a field smaller than the coercitive limit 


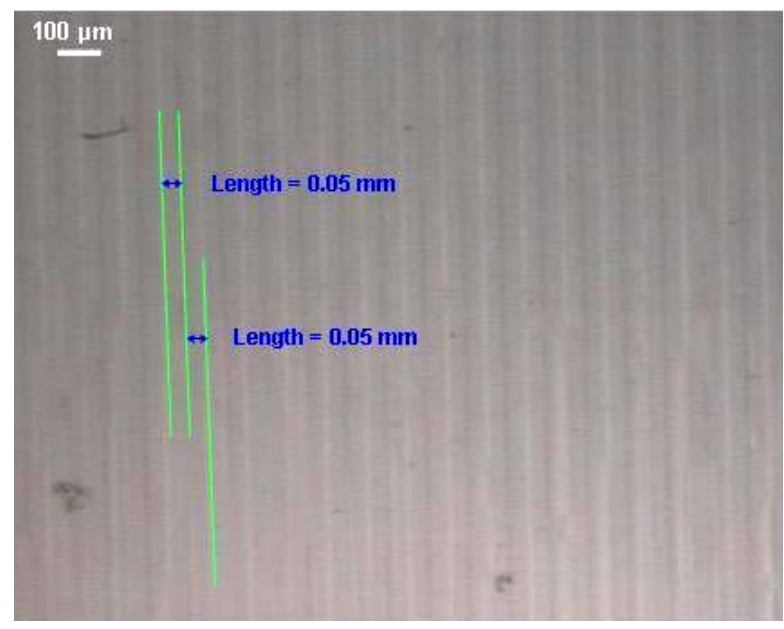

Figure 4. Optical verification of the poling efficiency (a) case of a $100 \mu \mathrm{m}$ period device, evidence of contrast changes between $Z^{+}$(native polarization) and $\mathrm{Z}^{-}$(switched polarization) domains (b) evidence of contrast modification in the vicinity of the poled domain walls at the poled strip edge (the whole plate is $\mathrm{Z}+$ oriented, the poled strips are $\mathrm{Z}^{-}$oriented)

\section{HIGH FREQUENCY DEVICES}

Different devices have been achieved and presented in [2] operating in the frequency range $20-50 \mathrm{MHz}$, showing a good predictability of their electrical response using period FEA. In this work, we have pushed one step further the fabrication of PPT to demonstrate their capability to operate in the intermediate frequency range $(50-500 \mathrm{MHz})$. In that matter, we have fabricated a new set of masks with poling periods (acoustic wavelengths) equal to 10,20 and $40 \mu \mathrm{m}$. Only the devices corresponding to the two last periods have been found to operate and allowed for electrical measurements. Figure 5 shows the admittance of a $20 \mu \mathrm{m}$ period PPT.

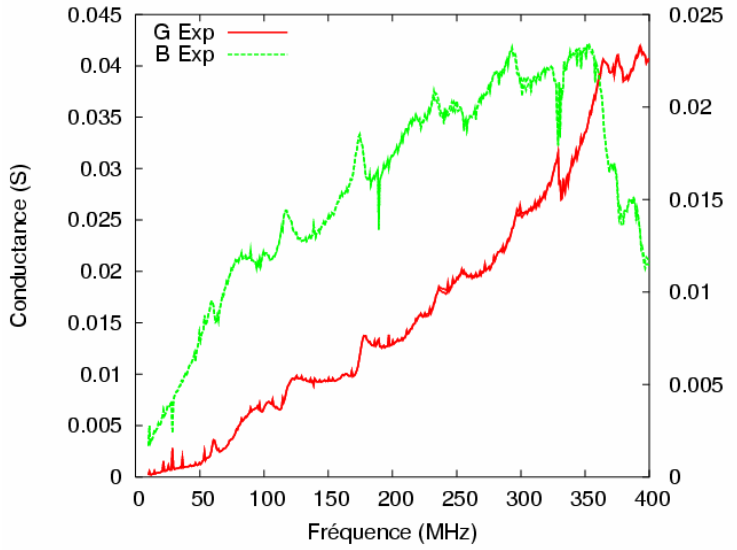

Figure 5. Experimental admittance of a $20 \mu \mathrm{m}$ period PPT

Although the response is somehow difficult to interpret (numerous parasitic bulk wave pollution), two strong contributions are pointed at 190 and $320 \mathrm{MHz}$ respectively. The first one was actually expected in the vicinity of $200 \mathrm{MHz}$ according to the above-presented first order analysis but we did not expect the one at $320 \mathrm{MHz}$ from the dispersion curves, even if they are far to be comprehensive regarding the operating conditions (wavelength-plate thickness ratio $m=0.04$ ). The same situation was pointed out for the $40 \mu \mathrm{m}$ wavelength device. We then have computed the harmonic admittance of the corresponding PPT structure and superimposed the obtained theoretical predictions with experimental measurements. This is reported in fig. 6 and 7 for the $20 \mu \mathrm{m}$ and $40 \mu \mathrm{m}$ period devices respectively.

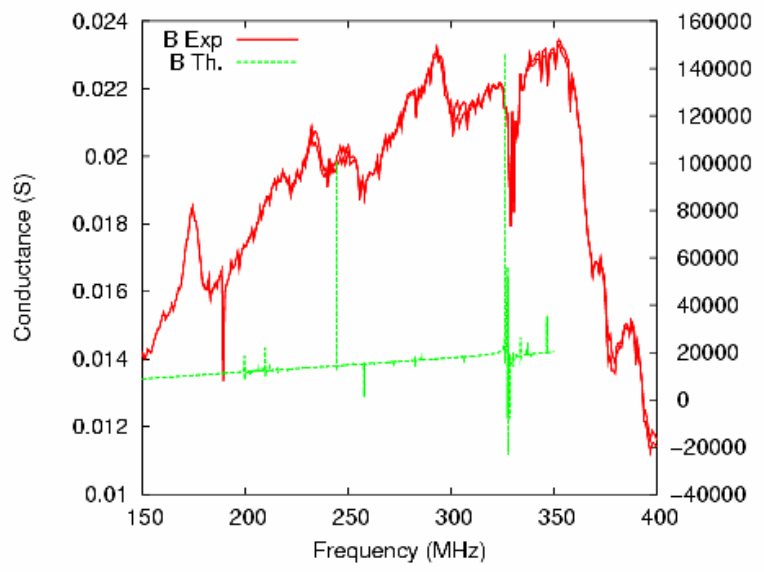

Figure 6. Theory/experiment assessment for the $20 \mu \mathrm{m}$ period PPT device

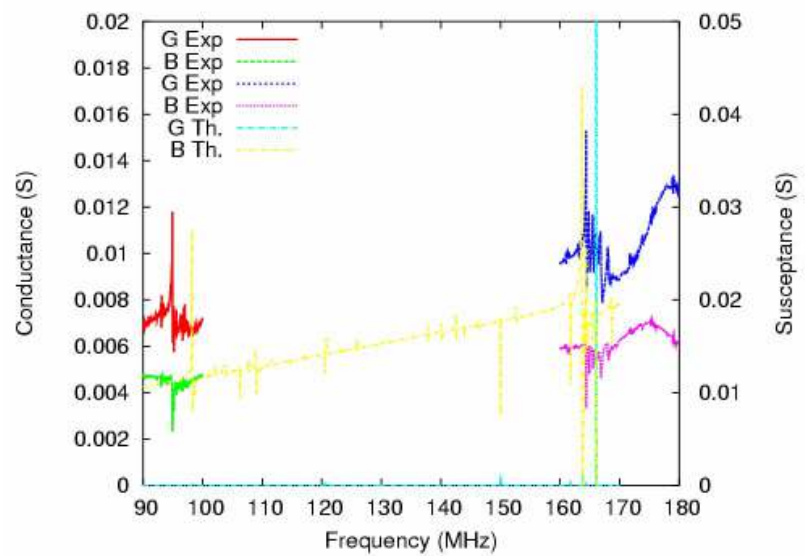

Figure 7. Theory/experiment assessment for the $40 \mu \mathrm{m}$ period PPT device. Experimental curves have been focused on the useful part to improve the plot readability

Even if the first modes are slightly shifted from theory to experiment, these plots point out the good agreement between both results. In a first order approximation, it is possible to calculate the equivalent phase velocity of each contribution, yielding values of 3800 and $6500 \mathrm{~m} . \mathrm{s}^{-1}$ respectively. Once again, the first value can be easily related to the asymptotic limit emphasized in fig. 1 for the very first Lamb wave contribution (i.e. Rayleigh wave velocity) but the second one approaches the longitudinal bulk wave velocity along $\mathrm{X}$ axis that does not clearly appear on the dispersion curve. As a consequence, we have computed the equivalent of a dispersion curve using our periodic FEA code and superimposed the predicted modes (the most important ones in terms of response 
amplitude for the sake of readability) with the Lamb wave dispersion curve of fig. 1 . This is reported in fig. 8 .

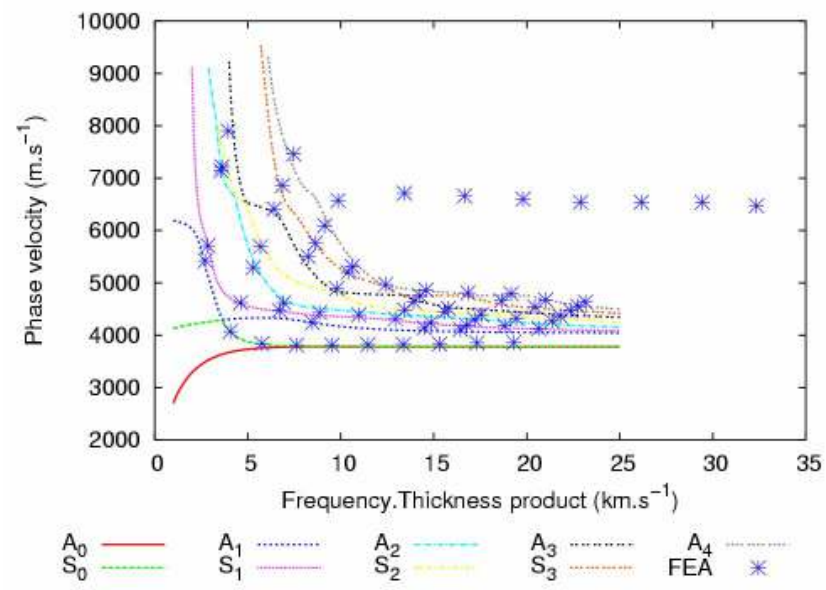

Figure 8. Superimposition of dispersion curves computed thanks to the Green's function approach and by periodic finite element analysis

This plot shows that a clear contribution of a quasilongitudinal mode propagating along the lateral direction of the plate arise for almost any frequency.thickness operating point. This contribution may be interpreted as the location where the high velocity Lamb waves strongly interacts, yielding significant changes in the group velocity between 6000 and $7000 \mathrm{~m} . \mathrm{s}^{-1}$. It can also be consider as a typical mode of the wave-guide, particularly favoured by the periodic poling of the plate. Note that such mode was not identified in previous work achieved on PZT [5] but it should certainly exist and should be emphasized along the same theoretical approach. Figure 9 shows the polarization of the mode near $165 \mathrm{MHz}$ for the $40 \mu \mathrm{m}$ period PPT device. Even if one can remark after a detailed analysis that the actual mode shape is a complex combination of shear, elliptical and piston-like displacements, the longitudinal polarization of the mode strongly predominates.

\section{CONCLUSION}

We have demonstrated the possibility to excite longitudinal modes using periodically poled transducer on $\mathrm{LiNbO}_{3} \mathrm{Z}$-cut. The velocity of these mode approaches 6500 $\mathrm{m} . \mathrm{s}^{-1}$, allowing for easily access intermediate and high frequency range without lithography breakthrough for the definition of the periodic grating required to build the devices. Although the numerous modes of our PPT clearly is a drawback for signal processing, it sounds that reaching very small wavelength-plate thickness ratio favor the spectral localization of those modes near asymptotical limits, corresponding to the Rayleigh wave and to the longitudinal mode along the plate surface. Although efforts have to be paid for improving the signal/noise ratio of the device response and to fully characterize the modes, these results encourage us to try and demonstrate the capability of PPT to actually achieve signal processing operations.

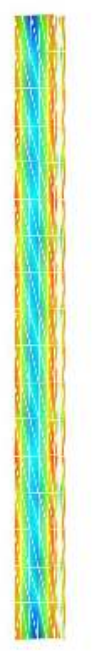

(a)

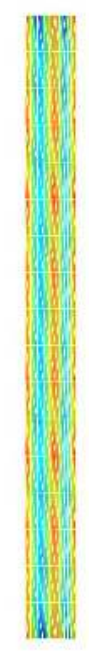

(b)

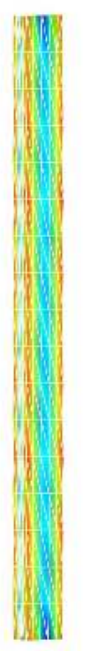

(c)

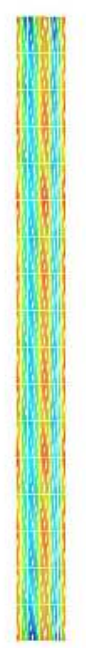

(d)
Figure 9. Sequence of dynamic vibration of the $40 \mu \mathrm{m}$ period PPT operating near $165 \mathrm{MHz}$, iso values of displacement along the plate radial dimension (a) $t=0$ (b) $t=1 /(4 . f)$ (c) $t=1 /(2 . f)$ (d) $t=3 /(4 . f)$

\section{REFERENCES}

[1] A. K. Sarin Kumar, P. Paruch, J.-M. Triscone, W.Daniau, S Ballandras, L. Pellegrino, D. Marré, T. Tybell, A High Frequency Surface Acoustic Wave Device Based on Thin Film Piezoelectric Interdigital Transducers, Appl. Phys. Let., Vol. 85, p.1717, 2004

[2] E. Courjon, N. Bodin, G. Lengaigne, L. Gauthier Manuel, W. Daniau, S. ballandras, Fabrication of periodically poled domain transducers on $\mathrm{LiNbO}_{3}$, Proc. of the IEEE IFCS, 2006

[3] S. Ballandras, A. Reinhardt, A. Khelif, M. Wilm, V. Laude, W. Daniau, V. Blondeau-Pâtissier, "Theoretical analysis of damping effects of guided elastic waves at solid/fluid interfaces", Journal of Applied Physics. 2006.99.054907

[4] L. E. Myers, R. C. Eckardt, M. M. Fejer, R. L. Byer, W. R. Bosenberg, J. W. Pierce, "Quasi-phase-matched optical parametric oscillator", JOSA B, Vol. 12, Issue 11, pp. 2102-2116, 1995

[5] S. Ballandras, W. Daniau, B. Gautier*, D. Hauden, M. Wilm, V. Laude, V. Ruch, S.Flambart, A novel surface wave transducer based on periodically poled piezoelectric domain, Proc of the IEEE IFCS, 2003 